PROCEEDINGS OF THE

AMERICAN MATHEMATICAL SOCIETY

Volume 30, No. 1, September 1971

\title{
ON THE ARENS PRODUCT AND ANNIHILATOR ALGEBRAS
}

\section{PAK-KEN WONG}

\begin{abstract}
The purpose of this paper is to generalize two results in a recent paper by B. J. Tomiuk and the author. Let $A$ be a $B^{*}$ algebra and $M(A)$ the algebra of double centralizers of $A$. We show that $A$ is a dual algebra if and only if $M(A)$ coincides with $A^{* *}$. We also obtain that if $A$ is an annihilator $A^{*}$-algebra, then $\pi_{A}(A)$ is a two-sided ideal of $A^{* *}$.
\end{abstract}

1. Notation and preliminaries. Notation and definitions not explicitly given are taken from Rickart's book [9].

For any subset $E$ of a Banach algebra $A$, let $l(E)$ and $r(E)$ denote the left and right annihilators of $E$ in $A$, respectively. Then $A$ is called an annihilator algebra if, for every closed left ideal $M$ and for every closed right ideal $N$, we have $r(M)=(0)$ if and only if $M=A$ and $l(N)=(0)$ if and only if $N=A$. If $M=l(r(M))$ and $N=r(l(N))$, then $A$ is called a dual algebra.

Let $A$ be a Banach algebra, $A^{*}$ and $A^{* *}$ the conjugate and second conjugate spaces of $A$, respectively. The Arens product on $A^{* *}$ is defined in stages according to the following rules (see [1]). Let $x$, $y \in A, f \in A^{*}, F, G \in A^{* *}$.

(a) Define $f \circ x$ by $(f \circ x)(y)=f(x y)$. Then $f \circ x \in A^{*}$.

(b) Define $G \circ f$ by $(G \circ f)(x)=G(f \circ x)$. Then $G \circ f \in A^{*}$.

(c) Define $F \circ G$ by $(F \circ G)(f)=F(G \circ f)$. Then $F \circ G \in A^{* *}$.

$A^{* *}$ with the Arens product $\circ$ is denoted by $\left(A^{* *}, \mathrm{\circ}\right)$.

Let $A$ be a semisimple Banach algebra. A pair $\left(T_{1}, T_{2}\right)$ of operators from $A$ to $A$ is called a double centralizer on $A$ provided that $x\left(T_{1} y\right)$ $=\left(T_{2} x\right) y$ for all $x, y$ in $A$. It has been shown that $T_{1}$ and $T_{2}$ are continuous linear operators on $A$ such that $T_{1}(x y)=\left(T_{1} x\right) y$ and $T_{2}(x y)$ $=x\left(T_{2} y\right)$ for all $x, y$ in $A$. The set $M(A)$ of all double centralizers on $A$ is a Banach algebra with identity and $A$ can be identified as a subalgebra of $M(A)$ (see [3]).

In this paper, all algebras and spaces under consideration are over the complex field $C$.

2. Double centralizers of $B^{*}$-algebras. Let $A$ be a $B^{*}$-algebra and $\left(A^{* *}, \mathrm{\circ}\right)$ its second conjugate space with the Arens product. It is

Received by the editors October 23, 1970.

AMS 1969 subject classifications. Primary 4650; Secondary 4655.

Key words and phrases. Dual $B^{*}$-algebra, Arens product, double centralizer, annihilator algebra, minimal idempotent, group algebra of a compact group. 
well known that $A$ is Arens regular and $\left(A^{* *}, 0\right)$ is a $B^{*}$-algebra (see $\left[4\right.$, p. 869, Theorem 7.1]). Since $A$ has an approximate identity, $A^{* *}$ has an identity $I$ by $[4$, p. 855 , Lemma 3.8]. Let $M(A)$ be the algebra of double centralizers of $A$. By [3, p. 81, Theorem 2.11], $M(A)$ is a $B^{*}$-algebra with identity.

Lemma 2.1. Let $A$ be a $B^{*}$-algebra. Then $M(A)$ is isometrically *-isomorphic to a subalgebra of $\left(A^{* *}, \mathrm{O}\right)$.

Proof. Let $\pi$ be the canonical mapping of $A$ into $A^{* *}$ and let

$$
Q=\left\{F \in A^{* *}: \pi(x) \circ F \text { and } F \circ \pi(x) \in \pi(A) \text { for all } x \in A\right\} \text {. }
$$

It is clear that $Q$ is a closed *-subalgebra of $A^{* *}$ containing the identity element $I$ of $A^{* *}$. Let $T=\left(T_{1}, T_{2}\right) \in M(A)$ and let $\left\{x_{\alpha}\right\}$ be an approximate identity of $A$. Since $\left\{\pi\left(T_{1} x_{\alpha}\right)\right\}$ is bounded, by Alaoglu's Theorem it has weak limit points in $A^{* *}$. Let $T_{1}^{\prime} \in A^{* *}$ be a weak limit point of $\left\{\pi\left(T_{1} x_{\alpha}\right)\right\}$. Since $\left(T_{1} x_{\alpha}\right) x=T_{1}\left(x_{\alpha} x\right)$, we have

$$
\left(T_{1}^{\prime} \circ \pi(x)\right)(f)=f\left(T_{1} x\right)=\pi\left(T_{1} x\right)(f),
$$

for all $f \in A^{*}$ and $x \in A$. Since by Goldstine's Theorem $\pi(A)$ is weakly dense in $A^{* *}$, it follows from (2.1) and the semisimplicity of $A^{* *}$ that $T_{1}^{\prime}$ is unique. Similarly $\left\{\pi\left(T_{2} x_{\alpha}\right)\right\}$ has a unique weak limit point $T_{2}^{\prime}$ such that $\pi(x) \circ T_{2}^{\prime}=\pi\left(T_{2} x\right)$. Since $x\left(T_{1} y\right)=\left(T_{2} x\right) y$ for all $x$, $y \in A$, it follows that $\pi(x) \circ T_{1}^{\prime}=\pi\left(T_{2} x\right)$ and $T_{2}^{\prime} \circ \pi(x)=\pi\left(T_{1} x\right)$. Now let

$$
T^{\prime}=\frac{1}{2}\left(T_{1}^{\prime}+T_{2}^{\prime}\right) \quad\left(T=\left(T_{1}, T_{2}\right) \in M(A)\right) .
$$

Then easy calculations show that $\pi(x) \circ T^{\prime}=\pi\left(T_{2} x\right)$ and $T^{\prime} \circ \pi(x)$ $=\pi\left(T_{1} x\right)$ for all $x \in A$. Therefore $T^{\prime} \in Q$. Let $S=\left(S_{1}, S_{2}\right) \in M(A)$. Since $S T=\left(S_{1} T_{1}, T_{2} S_{2}\right)$, we have

$$
\pi(x) \circ(S T)^{\prime}=\pi\left(T_{2} S_{2} x\right)=\pi(x) \circ\left(S^{\prime} \circ T^{\prime}\right),
$$

and so $(S T)^{\prime}=S^{\prime} \circ T^{\prime}$. If $T^{\prime}=0$, then $\pi\left(T_{2} x\right)=\pi(x) \circ T^{\prime}=0$ and hence $T_{2}=0$. Similarly $T_{1}=0$ and therefore $T=\left(T_{1}, T_{2}\right)=0$. It is easy to see that the mapping $T \rightarrow T^{\prime}$ is an onto mapping. Therefore it is an isomorphism of $M(A)$ onto $Q$. Since $M(A)$ and $Q$ are $B^{*}$ algebras, by $[7$, p. 395 , Theorem B $] T \rightarrow T^{\prime}$ is a ${ }^{*}$-isomorphism and therefore it is isometric by [6, p. 7, Proposition (1.3.7)] and [6, p. 16, Proposition (1.8.1)]. This completes the proof.

By Lemma 2.1, $M(A)$ can be considered as a subalgebra of $A^{* *}$.

TheOrem 2.2. Let $A$ be a $B^{*}$-algebra. Then $M(A)=A^{* *}$ if and only if $A$ is a dual algebra. 
Proof. This follows immediately from Lemma 2.1 and [10, p. 533, Theorem 5.1].

Let $A$ be a dual commutative $B^{*}$-algebra. Then $A^{* *}=A^{\prime \prime}$ by Theorem 3.2 in [11]. Hence Theorem 2.2 generalizes [10, p. 532, Theorem 4.2(3)].

3. The Arens product and annihilator algebras. In this section, unless otherwise stated, $A$ will be a semisimple Banach algebra with norm $\|\cdot\|$ which is a dense subalgebra of a semisimple Banach algebra $\mathfrak{A}$ with norm $|\cdot|$. Further $A$ and $\mathfrak{A}$ have the following properties:

(3.1) There exists a constant $k$ such that $k\|x\| \geqq|x|$ for all $x \in A$, i.e., $\|\cdot\|$ majorizes $|\cdot|$.

(3.2) Every proper closed left (right) ideal in $\mathfrak{A}$ is the intersection of maximal modular left (right) ideals in $\mathfrak{A}$.

(3.3) $A$ is an annihilator algebra.

If $A$ is an $A^{*}$-algebra and $\mathfrak{A}$ is the completion of $A$ in an auxiliary norm, then (3.1) and (3.2) automatically hold (see [6, p. 48, Théorème 2.9.5(iii)] and [9, p. 187, Corollary (4.1.16)]).

Notation. For any subset $E$ of $A, \mathrm{cl}_{A}(E)$ (resp. $\mathrm{cl}(E)$ ) will denote the closure of $E$ in $A$ (resp. $\mathfrak{l}$ ).

Let $S$ be the socle of $A$. Then $S$ is dense in $A$ by $[9$, p. 100, Corollary (2.8.16)]. Since $S$ is contained in the socle of $\mathfrak{A}$, it follows from $[2$, p. 571, Theorem 5.2] and (3.2) that $\mathfrak{A}$ is a dual algebra. Let $e$ be a minimal idempotent of $A$. Then $B=\operatorname{cl}_{A}(A e A)$ and $\mathfrak{B}=\operatorname{cl}(\mathfrak{A} e \mathfrak{A})$ are topologically simple, semisimple annihilator and dual algebras respectively (see $[9$, p. 100]). Let $I=A e$ and $\Im=\mathscr{A} e$. By $[9$, p. 67, Theorem (2.4.12)], $B$ and $\mathfrak{B}$ can be considered as operator algebras on $I$ and $\Im$, respectively.

Lemma 3.1. If $E$ is a proper closed subspace in $I$, then $E=\mathrm{cl}(E)$ is a proper closed subspace in $\Im$.

Proof. Let $R=\{T \in B: T(I) \subset E\}$. Since $E$ is a proper subspace in $I$, it follows easily from [9, p. 101, Lemma (2.8.20)] that $R$ is a proper closed right ideal in $B$. Let $\Re=\operatorname{cl}(R)$. Then $\Re$ is a proper closed right ideal in $\mathfrak{B}$. Since $\mathfrak{B}$ is a dual algebra, by $[9$, p. 105, Corollary (2.8.25)], we have $\Re=\left\{\mathfrak{I} \in \mathfrak{B}: \mathfrak{T}(\Im) \subset \mathscr{E}^{\prime}\right\}$, where, $\mathfrak{E}^{\prime}$ is a proper closed subspace in $\Im$. Since $B$ and $\mathscr{B}$ contain all operators with finite rank on $I$ and $\Im$, it follows easily that $E \subset \mathcal{F}^{\prime}$ and so $\mathbb{E} \subset \mathbb{F}^{\prime}$. Therefore (E) is a proper closed subspace in $\Im$.

Let $\pi_{A}$ (resp. $\pi$ ) be the canonical mapping of $A$ into $A^{* *}$ (resp. $\mathfrak{A}$ into $\left.\mathfrak{I}^{* *}\right) . A^{* *}$ and $\mathfrak{I}^{* *}$ with the Arens product will be denoted by $\left(A^{* *}, \circ\right)$ and $\left(\mathfrak{H}^{* *}, *\right)$. 
Lemma 3.2. If $\pi(\mathfrak{H})$ is a two-sided ideal of $\left(\mathfrak{U}^{* *}, *\right)$ then $\pi_{A}(A)$ is a two-sided ideal of $\left(A^{* *}, 0\right)$.

Proof. Let $e$ be a minimal idempotent of $A$ and let $X_{1}$ and $X_{2}$ be the normed spaces $(A e,\|\cdot\|)$ and $(A e,|\cdot|)$, respectively. The identity mapping from $X_{1}$ onto $X_{2}$ is denoted by $U$. It follows easily from Lemma 3.1 and (3.1) that $U$ maps maximal closed subspaces of $X_{1}$ to maximal closed subspaces of $X_{2}$. Similarly the inverse of $U$ has the same property. Therefore by $[8$, p. 246 , Lemma B], $\|\cdot\|$ and $|\cdot|$ are equivalent on $A e$. It follows easily that $A e=\mathfrak{A} e$. Therefore we can define the linear functional $e \cdot f$ on $\mathfrak{A}$ by

$$
(e \cdot f)(x)=f(x e) \quad\left(x \in \mathfrak{A}, f \in A^{*}\right) .
$$

Since $|(e \cdot f)(x)| \leqq k_{e}\|f\||x|$ for some constant $k_{e}$ (depending on $e$ ), $e \cdot f \in \mathfrak{A}^{*}$. Let $T$ be the mapping on $\pi(A e)$ into $A^{* *}$ given by

$$
(T(\pi(y e)))(f)=\pi(y e)(e \cdot f) \quad\left(y \in A, f \in A^{*}\right) .
$$

Since $T(\pi(y e))=\pi_{A}(y e)$, it follows that $T$ is a one-one mapping of $\pi(A e)$ onto $\pi_{A}(A e)$. For each $g \in \mathfrak{Q}^{*}$, let $g_{A}$ be the restriction of $g$ to $A$. Then by (3.1), $g_{A} \in A^{*}$. For each $F \in A^{* *}$, let $F^{\prime}$ be the linear functional on $\mathfrak{A}^{*}$ defined by

$$
F^{\prime}(g)=F\left(g_{A}\right) \quad\left(g \in \mathfrak{A}^{*}\right) .
$$

Then $F^{\prime} \in \mathfrak{A}^{* *}$ and hence by assumption, $F^{\prime} * \pi(e) \in \pi(\mathfrak{A} e)=\pi(A e)$. Easy calculations show that

$$
\left(T\left(F^{\prime} * \pi(e)\right)\right)(f)=\left(F^{\prime} * \pi(e)\right)(e \cdot f)=\left(F \circ \pi_{A}(e)\right)(f),
$$

for all $f \in A^{*}$. Hence $T\left(F^{\prime} * \pi(e)\right)=F \circ \pi_{A}(e)$ and so $F \circ \pi_{A}(e)$ $\in \pi_{A}(A e) \subset \pi_{A}(A)$. Since $A$ has dense socle, it follows easily that $A^{* *} \circ \pi_{A}(A) \subset \pi_{A}(A)$. Similarly we can show that $\pi_{A}(A) \circ A^{* *}$ $\subset \pi_{A}(A)$. This completes the proof.

The following result generalizes a part of $[10$, p. 533, Theorem 5.1].

TheOREM 3.3. Let $A$ be an annihilator $A^{*}$-algebra. Then $\pi_{A}(A)$ is a two-sided ideal of $\left(A^{* *}, 0\right)$.

Proof. Let $\mathfrak{A}$ be the completion of $A$ in an auxiliary norm. Since $\mathfrak{A}$ is a dual $B^{*}$-algebra, by $[10$, p. 533 , Theorem 5.1$], \pi(\mathfrak{A})$ is a twosided ideal of $\left(\mathfrak{A}^{* *}, *\right)$. Therefore by Lemma $3.2, \pi_{A}(A)$ is a two-sided ideal of $\left(A^{* *}, \mathrm{o}\right)$.

The following corollary generalizes a result by Civin (see $[5, \mathrm{p}$. 163, Theorem 2.4]). 
CoRollary 3.4. Let $A$ be the group algebra of a compact group. Then $\pi_{A}(A)$ is a two-sided ideal of $\left(A^{* *}, 0\right)$.

Proof. It is well known that $A$ is a dual $A^{*}$-algebra. Therefore the desired result follows from Theorem 3.3.

\section{REFERENCES}

1. R. Arens, The adjoint of a bilinear operation, Proc. Amer. Math. Soc. 2 (1951), 839-848. MR 13, 659.

2. B. A. Barnes, Modular annihilator algebra, Canad. J. Math. 18 (1966), 566578. MR 33 \#2681.

3. R. C. Busby, Double centralizers and extensions of $C^{*}$-algebras, Trans. Amer. Math. Soc. 132 (1968), 79-99. MR 37 \#770.

4. P. Civin and B. Yood, The second conjugate space of a Banach algebra as an algebra, Pacific J. Math. 11 (1961), 847-870. MR 26 \#622.

5. P. Civin, Ideals in the second conjugate algebra of a group algebra, Math. Scand. 11 (1962), 161-174. MR 27 \#5139.

6. J. Dixmier, Les $C^{*}$-algèbres et leurs représentations, Cahiers Scientifiques, fasc. 29, Gauthier-Villars, Paris, 1964. MR 30 \#1404.

7. L. T. Gardner, On isomorphisms of $C^{*}$-algebras, Amer. J. Math. 87 (1965), 384396. MR 31 \#3883.

8. G. W. Mackey, Isomorphisms of normed linear spaces, Ann. of Math. (2) 43 (1942), 244-260. MR 4, 12.

9. C. E. Rickart, General theory of Banach algebras, University Series in Higher Math., Van Nostrand, Princeton, N. J., 1960. MR 22 \#5903.

10. B. J. Tomiuk and P. K. Wong. The Arens product and duality in $B^{*}$-algebras, Proc. Amer. Math. Soc. 25 (1970), 529-535.

11. P. K. Wong, The Arens product and duality in $B^{*}$-algebras. II, Proc. Amer. Math. Soc. 27 (1971), 535-538.

McMaster University, Hamilton, Canada 